\title{
Las sombras de la energía solar fotovoltaica
}

\section{Análisis de los impactos negativos de su aprovechamiento en el medio ambiente}

\section{Resumen:}

Vivimos en una época en la que se demonizan las fuentes de "energías convencionales" debido a los efectos negativos que las misma tienen sobre el "medioambiente" o los accidentes sufridos en su explotación, y abrazamos sin ningún tipo de prejuicio y rápidamente las "energías renovables" sin hacernos muchas preguntas. Labúsqueda de soluciones o alternativas rápidas y socialmente aceptables a los problemas expuestos, nos llevan a no exigir traer a la superficie aquellas cosas no tan buenas que pueden tener las nuevas tecnologías, que tienen un gran abanico de puntos positivos frente a sus competidoras convencionales; $y$ que deben desarrollarse sin ocultar o esconder sus puntos negativos. En este trabajo se ponen en evidencia esas debilidades que presenta la energía solar fotovoltaica para que desde la visión ampliada nos permita exigir, interesados e involucrados en el futuro, que se profundicen los análisis en este tipo de tecnología y sus proyectos; para no vernos sorprendido por un impacto negativo que hoy sabemos o intuimos puede tener.

Palabras clave: energías convencionales - medioambiente - energías renovables.

\section{Summary:}

We are living on a time where the sources of "conventional energies" are being demonized due to the effects the latter are having on our "environment" or the accidents suffered thanks to its exploitation, and we embrace the "renewable energies" easily and without judgement; without asking ourselves too many questions or going beyond the basic. The search for quick and socially acceptable solutions or alternatives to the problems exposed, lead us not to

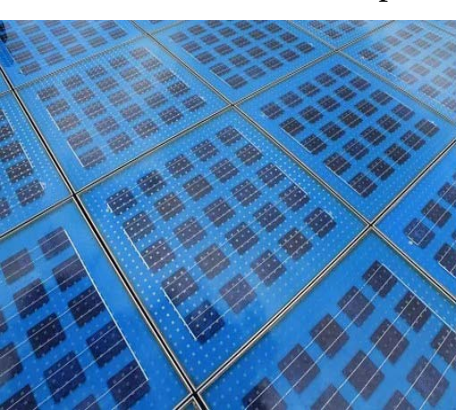
bring out those negative things that new technologies may have, these solutions have a wide range of positive points compared to its conventional competitors; but hiding the negative points is not the way the latter should develop. This work highlights those weaknesses that photovoltaic solar energy presents, so that from an expanded perspective it allows us to demand, as human beings interested and involved in the future, the analysis of this type of technology and its projects to be deepened; so as not to be surprised by a negative impact that we know or deduce it may have today.

Keywords: conventional energies - environment - renewable energies.

Diego Germán Lamas: dlamas@peuma-ig.com.ar https://orcid.org/0000-0001-6346-7319 Ingeniero Químico de la Universidad de Buenos Aires, con mas de 27 años de experiencia laboral en la industria manufacturera. Especialista en Calidad Industrial de la Universidad de San Martin/ INTI, Especialista en Organización y Dirección Empresaria de la Facultad de Ingeniería de la Universidad de Buenos Aires, cursando la Maestría en Gestión de la Energía en la Universidad Nacional de Lanús. Recibido:31/07/2020, Publicado: 15/10/2020. 


\section{Energía solar}

La energía del sol se ha usado desde el inicio de los tiempos y ha sido vital para todos los seres humanos y la naturaleza, gracias a esta es posible el proceso de fotosíntesis en las plantas.

\section{Objetivo del trabajo}

El objetivo de este trabajo es analizar en profundidad los impactos negativos de la tecnología solar fotovoltaica, desde la fabricación de sus componentes principales hasta la instalación de los sistemas completos y su posterior desarme y disposición al llegar al final de su vida útil.

\section{No se busca}

menospreciar el

aprovechamien-

to de esta fuente

de energía sino

analizar los efec-

tos desfavorables

que no son tan

ampliamente

comunicados

$y$ conocidos

comolos positi-

vos, para que los

mismo sean to-

mados en cuenta

al momento de

llevar adelante

un proyecto de

esta naturalezay

dar el puntapié

inicial para la incorporación de estos en

los Estudias de Impacto Ambiental (EIA).

1 El ciclo de vida de los activos nace desde la idea misma de realizar una actividad que involucrar activos en su desarrollo, pasa por las etapas de anteproyecto, proyecto, diseño, compra o manufactura, instalacion, prueba, puesta en marcha operación y mantenimiento, hasta su eventual reciclaje, descarte o disposición final.

Revista Perspectivas Metodológicas | Universidad Nacional de Lanús | ISSN 2618-4125 La energía solar se usa en todo el mundo y es cada vez más popular para generar electricidad, calentar y/o desalinizar agua.

Las llamadas células o celdas solares son dispositivos electrónicos que convierten a través del llamado efecto fotoeléctrico. ${ }^{2}$

\section{Capacidad instalada (MW)}

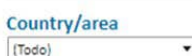

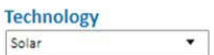$$
\begin{aligned}
& \text { Sub-technology } \\
& \text { Solar photovoltaic } \\
& \hline
\end{aligned}
$$$$
\text { III solar photovolta }
$$$$
300 \mathrm{~K}
$$

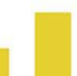

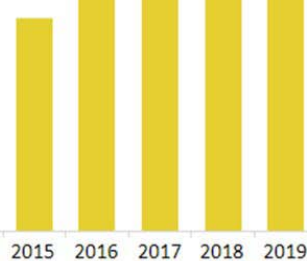

en día, la fotovoltaica, es una de las tecnologías de energía renovable de más rápido crecimiento y está lista para desempeñar un

2 El efecto fotoeléctrico es el fenómeno en el que las particulas de luz llamadas foton, impactan con os electrones de un metal arrancando sus átomos. El electrón se mueve durante el proceso, dado origen a una corriente eléctrica.
El costo de fabricación de paneles solares la luz solar directamente en electricidad

$$
\text { Show by }
$$

papel importante en la futura combinación global de generación de electricidad. se ha desplomado drásticamente en la última década, haciéndolos no solo asequibles sino a menudo la forma más barata de electricidad. Esto provoco el amplio desarrollo de esta tecnología, tal como lo podemos ver en el siguiente gráfico de Capacidad instalada.

Los paneles solares tienen una vida útil de aproximadamente 30 años.

La energía solar fotovoltaica, al igual que otras energías renovables, constituye, frente a los combustibles fósiles, una fuente inagotable, contribuye al autoabastecimiento energético y es menos perjudicial para el medio ambiente, evitando los efectos de su uso directo (contaminación atmosférica, residuos, etc.) y los derivados de su genera-

Esquemas de una instalación solar fotovoltaica

La energía eléctrica generada por un panel fotovoltaico se obtiene en forma de corriente continua y para poder utilizarla se pueden dar dos situaciones diferentes: que los consumidores trabajen con este tipo de corriente o que necesiten corriente alterna.
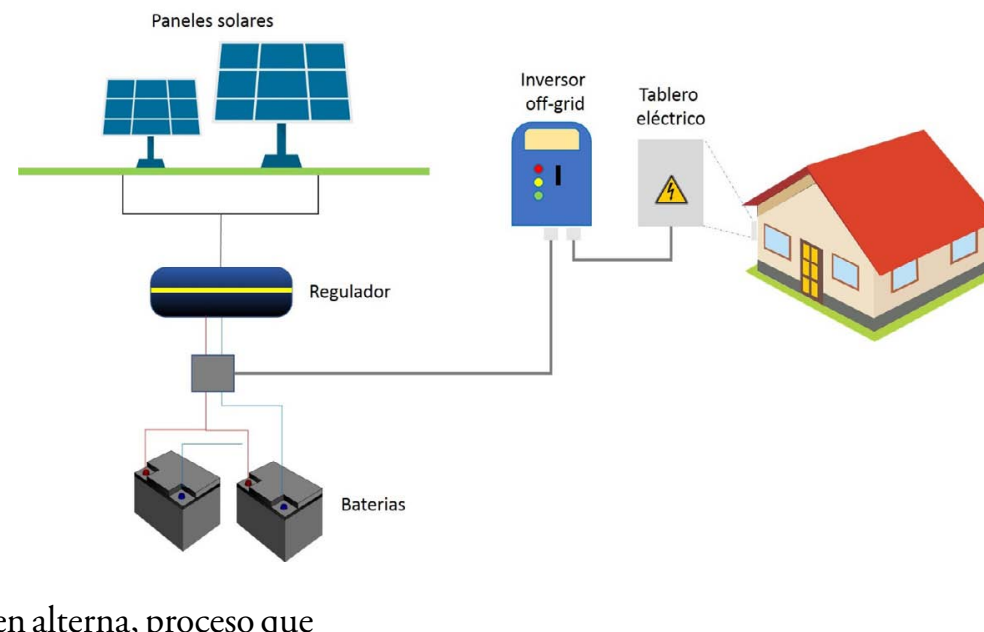
de corriente alterna es el más habitual bien para verterla a la red eléctrica o bien para autoconsumo, pero para ello será necesario realizar la transformación previa de corriente continua en alterna, proceso que

se realiza mediante un elemento llamado inversor. La otra opción es utilizar directamente la electricidad generada como corriente continua lo cual puede realizarse en algunos casos, como por ejemplo para iluminación de bajo consumo. En estos casos no sería necesario el inversor.

Además, en vez de utilizar la energía directamente también es posible al macenarla en una batería, con lo que se "guarda" hasta que realmente se necesita. De esta forma logramos aumentar la eficiencia del sistema.

Entonces, tenemos tres posibles formas de utilizar la energía generada: directamente como corriente continua, en forma de corriente alterna una vez transformada mediante un inversor, o almacenarla en una batería para su uso posterior.

Basado en los conceptos previos podemos definir tres tipos de instalaciones:

a) Instalaciones off-grid: Son los sistemas de instalaciones solares que están completamente desconectados de la red eléctrica. Estos sistemas son completamente independiente y se utilizan a menudo en áreas aisladas sin acceso a la red o en los casos donde se desea una completa independencia.

Revista Perspectivas Metodológicas | Universidad Nacional de Lanús | ISSN 2618-4125 
Un sistema fotovoltaico consta de tres partes principales:

1. Generador solar: compuesto por un conjunto de paneles fotovoltaicos, que captan la radiación solar y la transforman en corriente continua a baja tensión. (12, $24 \mathrm{o} 48 \mathrm{~V}$ )

2. Acumulador: Baterías que cumplen dos funciones: proveer potencia a la carga cuando no exista luz solar y amortiguar las variaciones de energía.

electricidad producida durante el día a la red de distribución, quien le paga por el ella, mientras que cuando no hay luz o ésta no es suficiente, el sistema no produce electricidad debido a que no hay un componente que almacene la energía (una batería) y debe consumir de la red de distribución, en el caso del generador-usuario, se plantea un balance entre la energía vendida y comprada a la red lo que optimiza el sistema eléctrico disminuyendo la energía eléctrica generada por sistemas convencionales.

3. Regulador de carga: Se utiliza estas unidades para evitar sobrecargas o descargas excesivas en el acumulador, lo cual produciría daños en el sistema y además se utiliza para que el panel siempre trabaje a la máxima eficiencia.

Y un cuarto elemento, en caso de necesitar corriente alterna.

4. Inversor: Componente en algunos sistemas fotovoltaicos que sirve para transformar la corriente continua en alterna.

b) Instalación on-grid: o conectada a la red, nos referimos a aquella que está conectada directamente con la red eléctrica local. Significa que durante las horas de uz del día el usuario consume la energía solar producida por su propia instalación, o también que esté actuando como generador-usuario (prosumidor) ${ }^{3}$ entregue la

3 La palabra prosumidor es un acrónimo formado por la fusión original de las palabras producto consumidor. El Prosumidor es un nuevo agente del mercado eléctrico que produce, consume, almacena y vende su propia energía eléctrica. Los prosumidores "Auto consumen" parte de electricidad que producen y venden el exceso fotovol taica que consume lo que produce es usada en casas pequeñas o en locales comerciales que solo necesitan electricidad durante el transcurso del día, sin embargo, para este tipo de instalación es indispensable tener acceso a la red eléctrica, por lo que no funciona en zonas aisladas.

Nota: En caso de contar con un sistema sobrante no utilizado para consumo propio.

c) Instalación parque solar: ${ }^{4}$ Son

a red. Pero cuando su producción es insuficiente, también compran energía de la red, lo que los convierte en productores y consumidores.

4 La diferencia entre un parque y una huerta solares está en el tamaño y en su carácter industrial o agrario. Un parque solar es una central solar se refiere a una instalación de gran tamańo, más industrial compuesta por varias plantas solare que requieren una sala de control centralizada transformadores de alta tensión. La huerta sola grupos de paneles solares, conectados generalmente a la red de distribución ${ }^{5}$ eléctrica, que generan potencias importantes responsables del gran aporte de electricidad al sistema, conectadas directamente a la red de transmisión. ${ }^{6}$ Los parques pueden estar conformados por un gran número de generadores fotovoltaicos individuales de diversas potencias. Normalmente los parques fotovoltaicos se construyen en zonas rurales y así permiten una alta producción de energía que puede ser usada para una gran cantidad de residencias o para fines industriales, $o$ para sumarse al sistema eléctrico. el caso de las centrales solares de potencia

Efectos de la energía solar

Los efectos generalmente descriptos de la energía solar fotovoltaica sobre los principales factores ambientales son los siguientes:

\section{- Clima}

La generación de energía eléctrica directamente a partir de la luz solar no requiere ningún tipo de combustión, por lo que no se produce polución térmica ni emisiones de $\mathrm{CO} 2$ que favorezcan el efecto invernadero.

- Geología

Las células
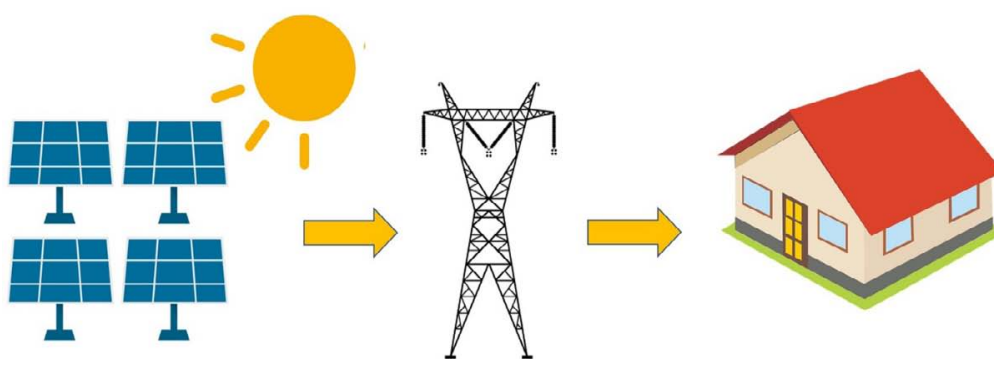
fabrican con silicio, elemento obtenido de la arena, muy abundante en la Naturaleza y del que no se requieren cantidades significativas. Por lo tanto, en la fabricación de acumulación puede entregar a la red el se refiere a instalaciones individuales de pequeño productores con la intención de producir energía a pequeña escala para venderla a la red eléctrica. Huerta solar tiene su origen en el carácter agripos, pastos o viñedos y porque metafóricamente se cultiva el sol para producir energía como otro cultivo más de la tierra.

5 La red de distribución es la parte del sistema de suministro eléctrico cuya función es el suministro de energía desde la subestación de distribució hasta los usuarios finale

6 La red de transmisión de energía eléctrica es la parte del sistema de suministro eléctrico con tituida por los elementos necesarios para llevar hasta los puntos de consumo y a través de grandes distancias, la energía eléctrica generada en las centrales eléctricas. cola porque se realizan encima de huertas, cam-

Al no producirse ni contaminantes, $\mathrm{ni}$ vertidos, ni movimientos de tierra, la incidencia sobre las características fisicoquímicas del suelo o su erosionabilidad es nula.

- Aguas superficiales y subterráneas

No se produce alteración de los acuíferos o de las aguas superficiales ni por consumo, $\mathrm{ni}$ por contaminación por residuos o vertidos.

- Flora y fauna

La repercusión sobre la vegetación es nula, y, al eliminarse los tendidos eléctricos, . 
se evitan los posibles efectos perjudiciales para las aves.

\section{- Paisaje}

Los paneles solares tienen distintas posibilidades de integración, lo que hace que sean un elemento fácil de integrar y armonizar en diferentes tipos de estructuras, minimizando su impacto visual. Esto es válido en pequeñas instalaciones domiciliarias o industriales, al tratarse de sistemas autónomos no se altera el paisaje con postes y líneas eléctricas, pero esto no es válido para las instalaciones más grandes, centrales solares de potencia que, por ejemplo, en argentina usan líneas de transmisión existentes, y en el caso de la planta solar de Cauchari, en la Provincia de Jujuy, a $4000 \mathrm{~m}$ de altura, requirió toda una línea de transmisión exclusiva.

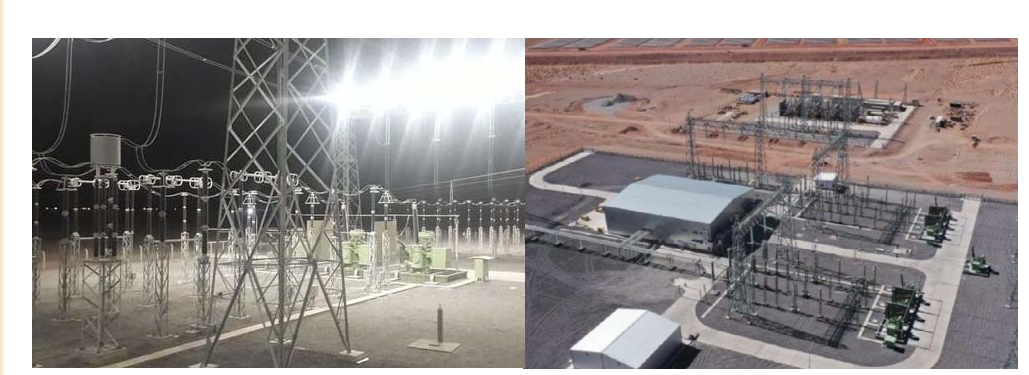

\section{- Ruidos}

El sistema fotovoltaico es absolutamente silencioso, lo que representa una clara ventaja frente a los generadores de motor en viviendas aisladas.

\section{- Medio social}

El suelo necesario para instalar un sistema fotovoltaico de dimensión media no representa una cantidad significativa como para producir un grave impacto. Además, en gran parte de los casos, se pueden integrar en los tejados de las viviendas.

"La perspectiva economicista de la energía solar fotovoltaica olvida un hecho

Revista Perspectivas Metodológicas | Universidad Nacional de Lanús | ISSN 2618-4125

4) Las fases de construcción y desmantelamiento de los parques solares son las más significativas en cuanto a las emisiones de gases, partículas, vertidos líquido, consumo de recursos, generación de residuos y afectación permanente del medio biótico. ${ }^{8}$

5) Gran cantidad de suelo ocupado por sus instalaciones (esencialmente en las grandes centrales fotovoltaicas).

7 https://www.ecologistasenaccion.org/10057/ impacto-ambiental/

$8 \mathrm{El}$ medio biótico lo constituyen todos los seres vivos del planeta, desde los organismos unicelulares hasta las grandes especies animales. Es lo que se conoce como biodiversidad.
2) Durante la pro-
ducción de los paneles solares se emite gran cantidad de gases de efecto invernadero y desechos tóxicos.

3) El principal consumo de agua

\section{Principales efectos negativos}

Los impactos ambientales negativos son aquellas alteraciones en el medioambiente que perjudican tanto el medio natural como la salud humana. Por tanto, las principales consecuencias son la contaminación del planeta (tierra, agua, y aire), la pérdida de biodiversidad y el incremento de enfermedades y problemas de salud.

\section{Alto consumo de energía}

Los módulos de silicio representan más del $90 \%$ del mercado fotovoltaico, pero las células solares de silicio, en sus dos variantes -mono cristalino (c-Si) y multi cristalino (mc-Si), tienen un problema: el elevado coste energético que supone obtener y purificar el material de partida. Esto se cuantifica mediante el tiempo de amortización energética (Energy Pay Back Time, EPBT), que es el tiempo que debe funcionar una célula solar para producir una cantidad de energía igual a la que se utilizó durante su proceso de fabricación e instalación.

de fabricación de los
En la gráfica de abajo se muestra el EPBT para distintas tecnologías fotovoltaicas en una localización con un nivel global de irradiación de $1925 \mathrm{kWh} / \mathrm{m}^{2} /$ año, característico del sur de Europa. Fuente: adaptado de Photovoltaics Report, julio 2017, Fraunhofer Institute for Solar Energy Systems (www.ise.fraunhofer.de)

Lo que se debe considerar también es que el mayor efecto por le energía utilizada para la producción de las celdas solares mono o policristalino de $S i$ es que, actualmente, usa energía convencional generada por centrales que utilizan fuentes convencionales y eso hay que sumarlo a los efectos de contaminación. En un futuro, cuando el porcentaje de electricidad generado por fuentes renovables sea muy alto ese efecto ira disminuyendo y finalmente desaparecerá

II. Gases de efecto invernadero (GEI) y desechos tóxicos

La obtención de silicio de grado metalúr gico es requerida en grandes cantidades para la industria del acero, siendo una pequeń proporción de este material la dedicada a la fabricación de las obleas de silicio. La emisión

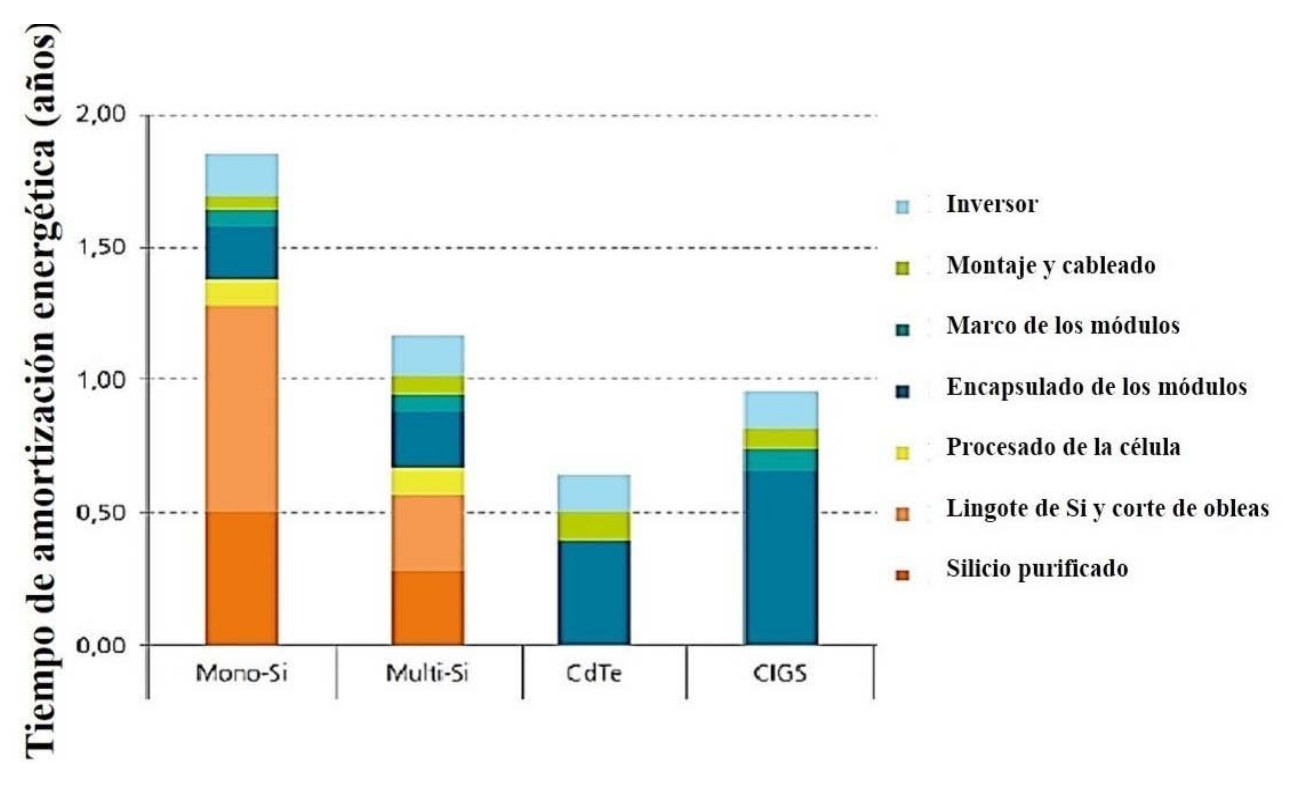

Revista Perspectivas Metodológicas | Universidad Nacional de Lanús | ISSN 2618-4125 
de polvo de sílice es uno de los inconvenientes de esta industria. La purificación del silicio implica el uso de materiales tales como xilano, mientras el dopado ${ }^{9}$ precisa utilizar pequeñas cantidades de compuestos tóxicos, tales como diborano y fosfina. También se precisa utilizar agentes agresivos, tales como el ácido sulfúrico.

\begin{tabular}{|l|l|l|l|l|}
\hline Tecnología & c-Si & $\begin{array}{c}\text { mc- } \\
\text { Si }\end{array}$ & CdTe & CGIS \\
\hline $\begin{array}{c}\text { Eficiencia Células } \\
(\%)\end{array}$ & 26,6 & 21,9 & 21,0 & 21,7 \\
\hline $\begin{array}{c}\text { Eficiencia módu- } \\
\text { los (\%) }\end{array}$ & 24,4 & 19,9 & 18,6 & 19,2 \\
\hline
\end{tabular}

Eficiencias record de células y módulos fotovoltaicos de las tecnologías principales, con datos actualizados a noviembre de 2017. https://blogs.publico.es/ignacio-mar

Con objeto de reducir el EPBT y los costos económicos de fabricación de los sistemas fotovoltaicos, en el mercado existen diversas alternativas, basadas en otros semiconductores.

a. CdTe: tanto el Cd (Cadmio) como el Te (Telurio) son elementos escasos en la corteza terrestre, por lo que la viabilidad de esta tecnología para la producción de paneles a muy gran escala (100 GW-1 TW) es cuestionable. Hoy en día es la tecnología con elEPBT más reducido. Para las células $\mathrm{de} C \mathrm{CT}$, se estima que se precisan menos de $200 \mathrm{~kg}$ de compuestos de Cadmio para producir $2 \mathrm{MW}$ anuales de células solares de esta tecnología.

b. CGIS (CuGaInSe2): de modo similar a la tecnología de $\mathrm{CdTe}$, algunos de los elementos químicos que utiliza son muy escasos (principalmente In y Se).

$$
\begin{aligned}
& \mathrm{Cu}=\text { Cobre } \\
& \mathrm{Ga}=\text { Galio } \\
& \mathrm{In}=\text { Indio } \\
& \mathrm{Se}=\text { Selenio }
\end{aligned}
$$

Las tecnologías descritas permiten obtener células solares con EPBT inferiores

9 Dopado: proceso intencional de agregar impurezas en un semiconductor extremadamente puro
con el fin de cambiar sus propiedades eléctricas.

Hay otras células solares basadas en otros semiconductores, fabricadas con silicio amorfo (a-Si) o con GaAs y compuestos afines (GaInP, GaInAs), donde As= Arsénico, $\mathrm{P}=$ Fosforo

En la actualidad, es ampliamente aceptado que la cantidad de gramos de $\mathrm{CO} 2$-equivalente es un buen indicador para realizar comparaciones del grado de contaminación asociado con los diversos métodos de generación eléctrica.

La siguiente figura muestra el rango de emisiones de GEI para distintas tecnologías $\mathrm{FV}$, estandarizadas para una irradiación de $1700 \mathrm{kWh} / \mathrm{m} 2 /$ año. Como se puede obser var, las tecnologías fotovoltaicas de CdTe se presentan entre las con menores rangos de emisiones de GEI entre las estudiadas.

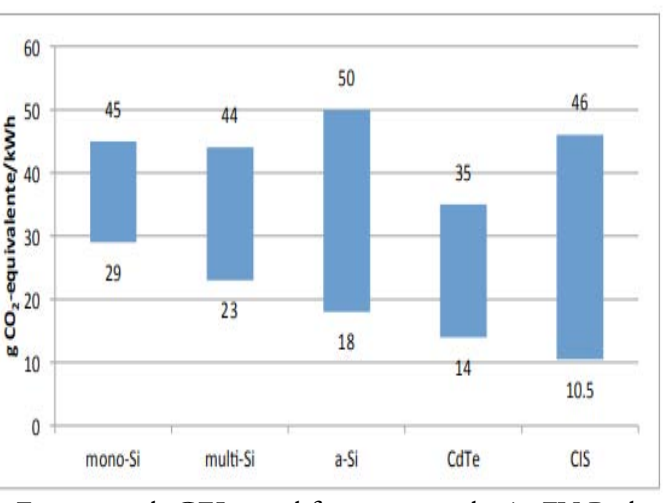

Emisiones de GEI para diferentes tecnologías FV. Radiación estandarizada en 1700 [kWh/m2/año]. Adaptado de Peng et al., 2013.
Muchos de los últimos paneles solares se están fabricados con un gas que contribuye con 17.000 veces más potencia que el dióxido de carbono al calentamiento global y que los científicos estiman que permanece en la atmósfera durante 550 años.

El trifluoruro de nitrógeno, $\mathrm{NF}_{3}$, se utiliza para limpiar los microcircuitos durante la fabricación de una serie de aparatos electrónicos modernos, entre ellos las televisiones planas, los iPhones, chips de ordenador.. y paneles extraplanos, la última (y más barata) generación de dispositivos solares fotovoltaicos.

"El NF3 tiene un posible efecto inver-
Como el sector calcula que sólo alrededor del $2 \%$ del $\mathrm{NF}_{3}$ sale a la atmósfera, la sustancia se vende como alternativa limpia a otras opciones que emiten más. Durante los últimos años, la Agencia de Protección Ambiental estadounidense ha fomentado su uso y no se consideró lo bastante peligroso como para hablar de él en el Protocolo de Kioto, con lo que se convirtió en un sustituto atractivo para empresas y países firmantes que querían reducir sus huellas contaminantes. nadero superior que... incluso las mayores centrales alimentadas con carbón del mundo", según un estudio publicado en junio de 2008 por investigadores de la Universidad de California. Como no está contemplado en Kioto, se han hecho pocos intentos de medirlo en la atmósfera.

Las primeras mediciones atmosféricas de gases de efecto invernadero generadas por los fabricantes de paneles solares alarmaron a muchos científicos del clima. La concentración de los gases producidos por el hombre, el trifluoruro de nitrógeno, fue cuatro veces mayor que los científicos habían previsto y un crecimiento del $11 \%$ al año.

Revista Perspectivas Metodológicas | Universidad Nacional de Lanús | ISSN 2618-4125
La industria de la energía solar representa el $5 \%$ de la demanda mundial de NF3, el resto procede principalmente de la fabricación de circuitos integrados. Sin embargo, durante la última década, la industria solar ha crecido en más del $45 \%$ anual.

En el proceso de fabricación de los paneles fotovoltaicos se usan numerosas sustancia peligrosas, muchas de las cuales se emplean para purificar y depurar la superficie semiconductora de los paneles. Estas sustancias químicas, similares a las usadas en la industria de los semiconductores, incluyen ácido clorhídrico, ácido sulfúrico, ácido nítrico, fluoruro de hidrógeno, 1,1,1-tricloroetano yacetona.

La cantidad y la sustancia en concreto que se usa depende el tipo de célula solar a fabricar, el grado de pureza que se necesita y el tamaño de la lámina de silicio. Los trabajadores también se exponen a riesgos por inhalación de polvo de silicio. Por ello, en el proceso de fabricación se deben garantizar unas condiciones laborales seguras para lo trabajadores y que los productos usado y residuos, se gestionan adecuadamente.

Los paneles fotovoltaicos de capa fina contienen un mayor número de sustancia tóxicas respecto a los paneles de silicio tradicionales. En su fabricación se emplean arseniuro de galio, di seleniuro de cobre-indio-galio, y teluro de cadmio. Si no se manejan y se desechan apropiadamente, estas sustancias químicas pueden ocasionar un serio problema de contaminación ambienta y amenazar la salud pública.

\section{Alto consumo de agua}

Los fabricantes de paneles y celdas utilizan una gran cantidad de agua para diversos fines, que incluyen la refrigeración, los procesos químicos y el control de la contaminación del aire generada en el proceso. 
La cantidad de agua utilizada para producir, instalar y operar los paneles fotovoltaicos es significativamente menor que la necesaria para enfriar las plantas termoeléctricas alimentadas por combustibles fósiles.

No obstante, el mayor desperdicio de agua se da durante la limpieza en la instalación yel uso. Los proyectos a escala comercial en un rango de 230 a 550 megavatios pueden requerir
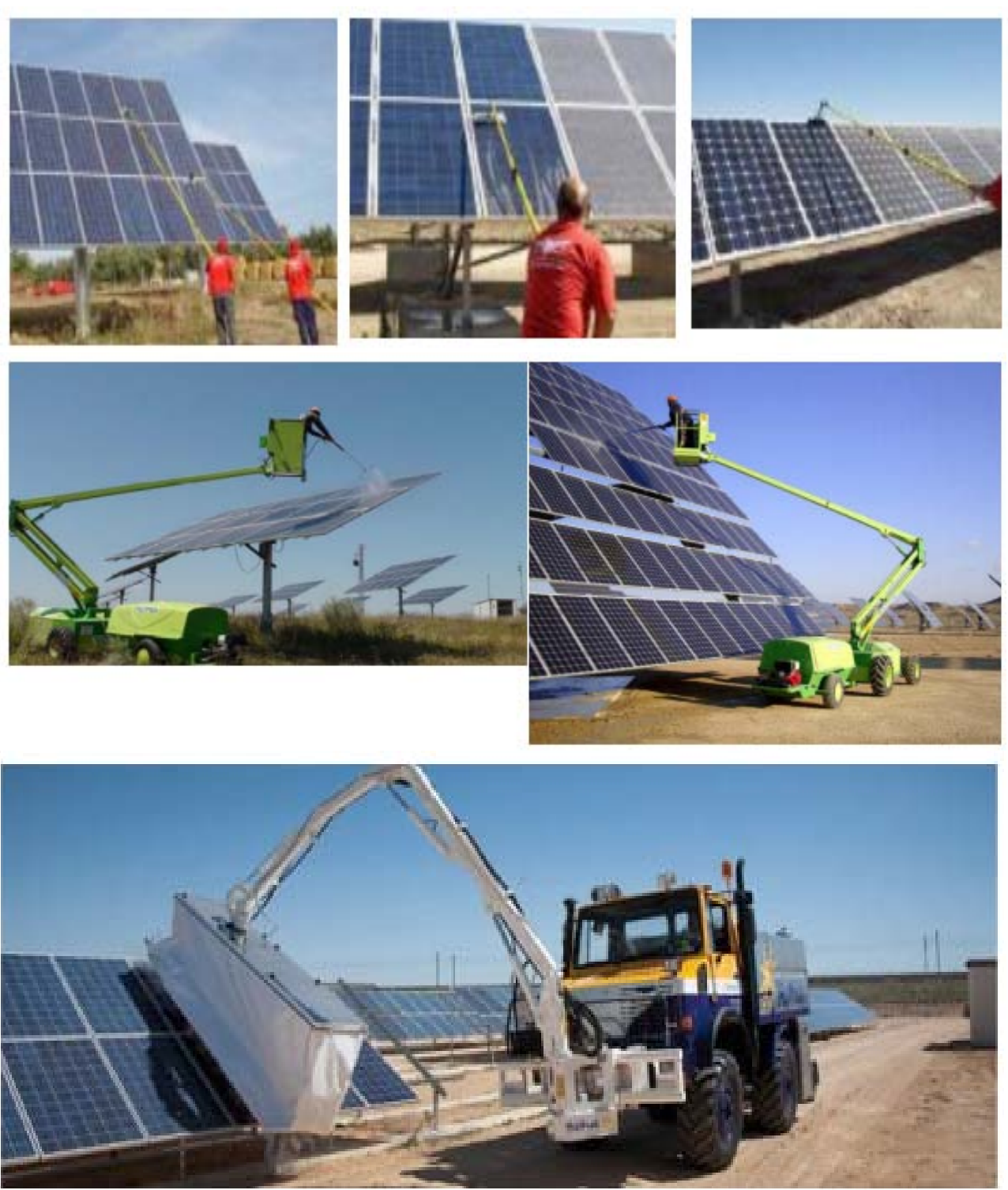

Revista Perspectivas Metodológicas | Universidad Nacional de Lanús | ISSN 2618-4125

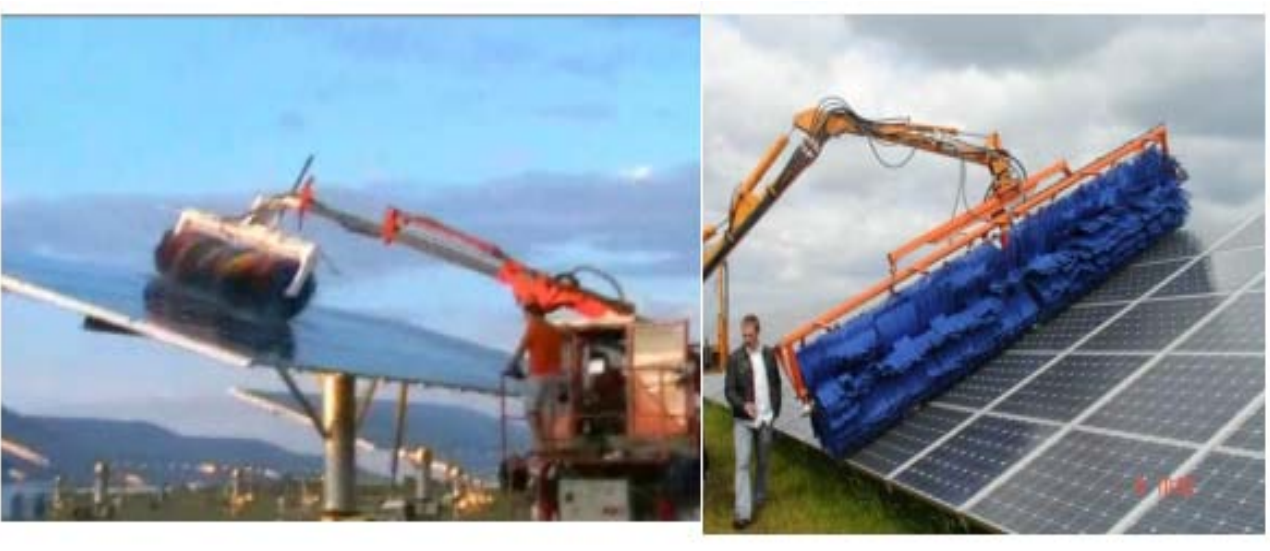

Pero pese a todolo antes dicho, un estudio realizado por la Universidad LUT de Finlandia, concluye que la energía solar fotovoltaica consume entre el $2 \%$ yel $15 \%$ del agua quelas plantas de carbón y energía nuclear utilizan para producir solo 1 MWh de energía.

IV. Fase de construcción y desmantelamiento

La generación de electricidad mediante ESFV requiere la utilización de grandes superficies colectoras y por tanto de una cantidad considerable de materiales para su construcción. La extracción, producción y transporte de estos materiales son los proceso que suponen un mayor impacto ambiental.

Las fases de construcción y desmantelamiento son los más significativos en cuanto a las emisiones de gases, partículas, vertidos líquidos, consumo de recursos, generación de residuos y afectación permanente del medio biótico. Estos factores afectan, esencialmente a las grandes centrales FV. En particular, la construcción de las vías de acceso y las edificaciones auxiliares causan los impactos más significativos sobre la vegetación, la fauna y el suelo. La implementación de este tipo de proyectos debe priorizar aquellas zonas de menor presencia de vegetación con el fin de minimizar los impactos.

La fabricación de un panel solar requiere también la utilización de materiales como aluminio (para los marcos), vidrio (como encapsulante), acero (para estructuras) etc. siendo estos componentes comunes con la industria convencional.

Finalmente se puede señalar la existencia de fuentes contaminantes relacionadas con la producción de ESFV aunque no sean debidas a la producción de paneles solares. Esta contaminación proviene de la fabricación de equipos tales como inversores, reguladores, estructuras de soporte, cable y especialmente acumuladores. Algunos de estos sistemas están presentes, necesariamente, en todas las instalaciones de ESFV haciendo así depender el análisis del tipo de instalación considerada.

\section{Alta utilización del suelo}

Uno de los principales argumentos esgrimidos en contra de la ESFV es la cantidad de suelo ocupado por sus instalaciones y/o la ocupación de espacio por parte de los paneles solares no integrados en la arquitectura, los movimientos ecologistas apuestan por un desarrollo prioritario de la ESFV integrada en la arquitectura y de un modo más simple, aprovechando la superficie d tejados y fachadas ya disponibles. Pero esto 
no es posible cuando hablamos de centrales solares de potencia.

Este argumento no es un inconveniente real para la implantación de este tipo de energía. La consideración de todos los factores que contribuyen a la ocupación del suelo (minería, construcciones etc.), sitúan, en este aspecto a la ESFV en un lugar parecido al de las centrales térmicas e incluso en un mejor lugar que a algunas de las tecnologías actuales para la obtención de energía.

Dependiendo de su localización, las plantas solares más grandes pueden provocar una degradación del suelo y pérdidas de hábitats. Las necesidades totales de suelo varían en función de la tecnología, la topografía de la zona y la intensidad de la radiación. En una instalación de solar fotovoltaica, las necesidades son de 2 ha por megavatio instalado en el caso de paneles solares policristalino y de $5 \mathrm{ha} / \mathrm{MW}$ para paneles de capa fina.

Si bien la ESFV precisa una cantidad de suelo mayor que otras energías renovables, ahorra espacio en comparación con los centrales de producción energética mediante carbón.

\begin{tabular}{|l|c|}
\hline $\begin{array}{c}\text { Cantidad de suelo ocupado (en m2) } \\
\text { por una instalación productora de 1 } \\
\text { GWh de energía durante } 30 \text { ańos para } \\
\text { varias tecnologías }\end{array}$ \\
\hline Tecnología & $\begin{array}{c}\text { Espacio (m2/GWh } \\
\text { en } 30 \text { años) }\end{array}$ \\
\hline Carbón & 3642 \\
\hline Térmica solar & 3561 \\
\hline Fotovoltaica & 3237 \\
\hline Eólica & 1335 \\
\hline Geotérmica & 404 \\
\hline \multicolumn{2}{|c|}{ https://www.ecologistasenaccion. } \\
org/10057/impacto-ambiental/ \\
\hline
\end{tabular}

El impacto sobre el suelo podría verse minimizado si se destinasen a la implantación de huertos solares aquellas áreas más degradadas o campos de cultivo abandonados por su baja productividad. Por supuesto, las instalaciones más pequeñas en hogares o industrias no ocasionan impacto alguno sobre el terreno al situarse normalmente en las cubiertas de las edificaciones existentes.

El estudio publicado por la revista científica PNAS (Solar energy development impacts on land cover change and protected areas, en 2015) señala la importancia de situar las plantas solares en lugares cuidadosamente pensados, de forma que no invadan terreno bajo protección medioambiental, que no amenacen ecosistemas naturales ni especies protegidas y que tampoco acaparen áreas dedicadas a otras actividades económicas.

Los autores del estudio denuncian que han observado daños medioambientale extensivos a causa de la ubicación descuidada de este tipo de instalaciones, "cuando simplemente con pensar prudentemente dónde situarlas puede aliviar ese daño". Lo impactos pueden ir desde un aumento de invasiones por parte de especies no nativas hasta el cambio en hábitats protegidos como respuesta a perturbaciones ambientales, igual que ocurre con el cambio climático.

En el estudio asegura que tenemos la información, la tecnología y la motivación económica para lograr una transición rápida y eficiente a un modelo energético solar, pero a cambio no sabemos lo suficiente sobre cómo los ecosistemas áridos, de los que los humanos dependemos para muchas cosas, pueden ser restaurados después de una agresión importante, con muy pocos casos de éxito en los que poder basarnos.

Solo para tomar conciencia de las magnitudes de las que se hablan, la superficie de la Ciudad Autónoma de Buenos Aires
(CABA) es de $203 \mathrm{~km}^{2} \mathrm{y}$ "La Gran Muralla China del Sol": la planta solar más grande

del mundo cubre más de 1.200 km2 y está ubicada en el desierto de Tengger.

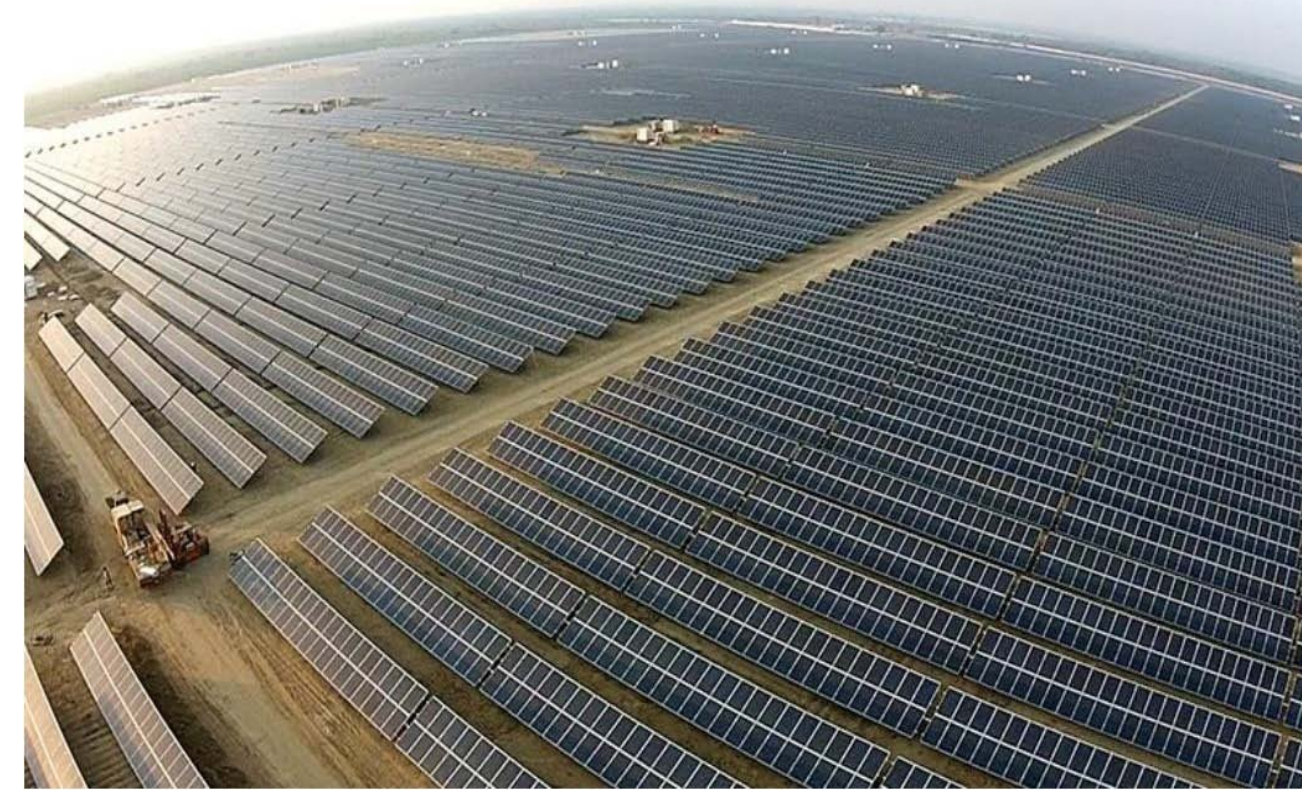

Conclusiones

La energía solar que algunas veces se conoce como limpia o amigable con el medio ambiente, y algunas veces se piensa que producen cero contaminaciones también tienen su impacto negativo durante su proceso de elaboración y posterior eliminación debido a que generan algunos contaminantes y emisiones a la atmosfera que pueden llegar a ser perjudiciales para los seres humanos, sin embargo, esta tecnología contamina en menor proporción que los combustibles convencionales.

Los potenciales impactos medioambientales asociados a la energía solar, como el uso del suelo y pérdida de hábitats, el uso de agua, así como el uso de materias prima peligrosas en la fabricación de paneles y otro componentes de las instalaciones solares, varían mucho en función de la tecnología empleada para aprovechar la energía del sol.
También el tamaño de una instalación juega un importante papel. Las instalacione solares pueden ser tan simples como un panel solar situado en la cubierta de una casa. Pero también es cierto que existen grandes huertos o explotaciones solares, cuyo impacto en el medio ambiente es lógicamente mucho mayor.

Se considera que los impactos medioambientales de la ESFV son ecológicamente asumibles, supuesto un correcto tratamiento o almacenaje de residuos y una correcta gestión del resto de los impactos ambientales.

La tecnología fotovoltaica avanza en el sentido de ir contrarrestando los efectos negativos como los comentados, po ejemplo, el pasar de las células de Si a las de CdTe mejoró el alto consumo de energía a costa de eficiencia, pero nuevos procesos, 
nuevos materiales, nuevo requerimiento hacen necesario no perder de vista estos procesos de mejora continua, a fin de que no aparezcan nuevos efectos a contrarrestar, como lo fue la generación de un nuevo GEI como el $\mathrm{NF}_{3}$ que no había sido considerado en el acuerdo de KIOTO, por lo cual su impacto no era medido.

Que la energía solar fotovoltaica provenga de una fuente inagotable y gratuita, no implica que no deba desarrollarse tomando todos los cuidados y recaudos requeridos, para que la intervención del hombre en su afán de aprovecharla rápidamente no magnifique los efectos negativos que la utilización de esta tecnología puede tener sobre el medio ambiente.

\section{Bibliografía}

—Guzmán Niño, C. A. (2017). - Análisis del impacto ambiental de diferentes tipos de paneles solares según los materiales utilizados y los componentes tóxicos generados. (Monografía para optar por el título de Especialista en Gestión Ambiental). Recuperado de http://repository.uamerica.edu.co/ bitstream/20.500.11839/7038/1/6325752017-II-GA.pdf

- Pascualino, J. Cabrera, C. Vanegas Chamorro M. (2015). "The environmental impacts of folic and solar energy implementation in the Colombian Caribe". Prospect, Vol 13, No 1, pp 68-75. Recuperado de http:// www.scielo.org.co/scielo.php?script=sci arttext\&pid=S1692-82612015000100008

-Departamento de energía solar fotovoltaica, Centro Nacional de Energías Renovables (CENER) y Área de energía y cambio climático de Fundación Chile (2013).

- Tecnología Fotovoltaica de CdTe de First Solar: Evaluación Medioambiental, de Salud y Seguridad. (Informe Final). Recuperado de http://www.firstsolar.com/-l media/First-Solar/Sustainability-Documents/
Sustainability-Peer-Reviews/ES-First-Solar-Peer-Review-CENER_FCh_Espanol Informe Final.ashx

- Hernandez,R.R.; Hoffacker, M.K.; Murphy-Mariscal, M.L.; Wu G.C. y Allen, M.F. (2015, 19 de Octubre). "Solar energy development impacts on land cover change and protected areas". PNAS (Proceedings of the National Academy od Sciences of the United States of America). Recuperado de https:// www.pnas.org/content/112/44/13579/

—Bellini, E. (2019, 12 Diciembre). "100\% renewables means $95 \%$ less water consumption for conventional power generation". PV Magazine. Recuperado de https://www. pv-magazine.com/2019/12/12/100-renewables-means-95-less-water-consumption-for-conventional-power-generation/

—Energías Renovables (2015, 27 de febrero). "Impacto medioambiental de la energía solar". Energias Renovables. Recuperado de http://www.energiasrenovablesinfo.com/ solar/impacto-medioambiental-energia-solar/ — Renovables (2007, 21 de junio). "[Fotovoltaica] Impacto ambiental”. Recuperado de https://www.ecologistasenaccion. org/10057/impacto-ambiental/ 